\title{
Social work supervision in child and family services: Developing a working theory of how and why it works
}

\author{
David Wilkins Cardiff University, Wales, United Kingdom
}

\begin{abstract}
INTRODUCTION: Does social work supervision work? Social work academics and others have argued repeatedly that we need to focus more attention on understanding whether and how supervision helps improve outcomes for people who use services. As things stand, we currently have little evidence either way-and although the absence of evidence is not evidence of absence, this is far from an ideal situation.

APPROACH: Taking inspiration from realist approaches to evaluation, this article sets out an initial working theory of social work supervision for child and family services, developed from an analysis of six significant reviews of the supervision literature. Each review was analysed to identify key contexts, mechanisms and outcomes for supervision.

CONCLUSION: Notable gaps within the theory are identified in relation to workers, outcomes for children and families and how supervision can promote a rights-based approach. The article concludes by arguing that this working theory offers the basis for future evaluative studies of supervision.
\end{abstract}

KEYWORDS: Supervision, social work, children and families, what works
It is widely accepted that the evidence base for supervision is relatively weak. No review or meta-analysis has ever found strong evidence that supervision consistently makes a difference for people who use services, and there is only relatively weak evidence that it makes a consistent difference for workers (Carpenter, Webb, \& Bostock, 2013). A Delphi study in 2015 identified that, amongst a group of international experts, the need to establish the evidence base for supervision, and particularly in relation to people who use services, was viewed as a pressing priority (Beddoe, Karvinen-Niinikoski, Ruch, \& Tsui, 2015). Despite these limitations, there remains a strongly held belief within the social work profession that high-quality supervision really does make a difference, not only for workers but for people who use services too. And while it is true that "a consensus is not an evidence-base" (Forrester et al., 2019, p. 3), it is also true that the absence of evidence is not evidence of absence.

In recent years, there has certainly been an increased focus on supervision within the social work academy, demonstrated not least by an increasing number of journal publications (O'Donoghue \& Tsui, 2015; Sewell, 2018). In the Aotearoa New Zealand Social Work journal alone, there have been 23 articles (including book reviews) published with supervision in the title in the past 10 years. (The significance of this journal's contribution in particular has been recognised internationally in Sewell, 2018.)
AOTEAROA NEW ZEALAND SOCIAL WORK 31(3), 7-19.

CORRESPONDENCE TO: David Wilkins WilkinsD3@cardiff.ac.uk 
In England, there has also been a concerted effort in practice to provide more effective supervisory support, particularly for early-career practitioners (Schraer, 2016). Similar efforts have been made elsewhere, as Rankine (2017) outlines in relation to Aotearoa New Zealand. Despite these well-intended and in many cases successful efforts the general picture remains one of concern about the managerial capture of supervision (Manthorpe, Moriarty, Hussein, Stevens, \& Sharpe, 2015; Wilkins, Forrester, \& Grant, 2017). Turner-Daly and Jack (2015) found that supervision sessions in England may focus on case-management to the exclusion of much else, while Baginsky et al. (2010) reported that senior managers often consider supervision to be a mechanism for performance-management, rather than a forum for support. In Aotearoa New Zealand, similar concerns have been expressed by Rankine (2017) and Moorhouse, Hay, and O'Donoghue (2014), with the suggestion that supervision functions in many cases as a mechanism for the surveillance of practice within a neoliberal context.

Yet, it is also true that more effective supervision is not only possible but is in some places flourishing. In a number of local authorities in England, there has clearly been much thought given to the purpose of supervision and thriving cultures of reflective and supportive approaches have been and are being developed (Lees, 2017). Bostock's work has been instrumental in helping to identify key components in the relationship between certain forms of group systemic supervision and the practice skills demonstrated by workers (Bostock, Forrester, Patrizo, Godfrey, \& Zonouzi, 2017; Bostock, Patrizo, Godfrey, Munro, \& Forrester, 2019). Davys, May, Burns, and O'Connell (2017) have also looked at how supervision is evaluated in practice by supervisors and supervisees in Aotearoa New Zealand. Research from America has helped identify how supervision can improve workers' knowledge of theory and practice models (Smith et al., 2007) and self- efficacy (Collins-Camargo \& Royse, 2010), as well as influencing job satisfaction and retention rates (Mor Barak, Travis, Pyun, \& Xie, 2009).

However, as we strive to prove that supervision works, it is important to consider (or not to forget) that simplistic conceptions of effectiveness are likely to be unhelpful. It is increasingly recognised, particularly within the realist tradition, that the evaluation of complex social interventions cannot simply ask "what works?" Instead, we need a more nuanced understanding of what works for who and how and why (Pawson \& Tilley, 2014). This is especially true when considering the complex interplay of values, ethics, power and culture that takes place within supervision (see Elkington, 2014). As Pawson and Tilley (2014) explain, when undertaking evaluations, we need to consider how human beings behave, decide and respond within social contexts, and to understand complexities such as motivation and inter-personal relationships. A parenting programme may work for a group of enthusiastic, motivated and voluntary attendees but is less likely to work for a group of demotivated and mandated attendees. For complex interventions, they work (or not) because the people involved in them behave in certain ways and make particular decisions - and it is these behaviours and decisions rather than the intervention per se which 'cause' the outcomes. This approach speaks directly to the importance of considering questions of culture and diversity, something long recognised within the supervision literature as being of paramount importance (Cashwell, Looby, \& Housley, 1997).

\section{Developing a working theory}

Realist evaluation "starts with a theory and ends with a more refined theory" (Currie, Chiarella, \& Buckley, 2019, p. 1322). The purpose of realist evaluation is to develop and test what is known as a programme 
theory (see Byng, Norman, \& Redfern, 2005; Byng, Norman, Redfern, \& Jones, 2008). This kind of mid-range theory seeks to describe how an intervention works. It includes a description of the wider conditions in which the intervention should be situated and the kinds of outcomes you can expect to achieve. It also describes the moderators that influence how the intervention works (Funnell \& Rogers, 2011). There is no one way to express a programme theory (Taylor-Powell \& Henert, 2008) but they will usually include inputs (e.g., staff, funding, training), outputs (e.g., meetings, advice and information) and outcomes (e.g., changes in behaviour and mindset). The aim of a programme theory is to provide a simplified model to communicate the key elements of an intervention (TaylorPowell \& Henert, 2008).

Theory development of this kind does not rely solely on existing empirical work. Indeed, given that realist evaluation starts with theory, this would not always be possible. Instead, the theory is developed from a range of sources, which may include existing empirical work, but can also involve conceptual and theoretical discussions and the experiences, expertise and views of people involved in the intervention. When these theories are developed based on existing literature, it is common to undertake consultation and empirical work as a natural development and testing process of the theory (see Shearn, Allmark, Piercy, \& Hirst, 2017). For this article, the programme theory has been developed based on six existing reviews of the supervision literature and in relation to the context of child and family social work specifically.

\section{Defining supervision}

As the purpose here is to develop a working theory of supervision for child and family social work, the following definition is used: "Supervision is a regular, planned, accountable process, which must provide a supportive environment for reflecting on practice and making well informed decisions using professional judgement and discretion" (British Association of Social Workers, 2011, p. 7). Other definitions are available and as noted by Sewell, "caution is needed [because] nomenclature is used differently" (2018, p. 253). Social work supervision is considered here specifically, as distinct from supervision more generally (for example, in psychotherapy or infant mental health work). Sewell's suggested definition by way of comparison is that supervision:

...is the relationship between supervisor and supervisee in which the responsibility and accountability for the development of competence, demeanour and ethical practice takes place. The supervisor is responsible for providing direction to the supervisee, who applies social work theory, standardized knowledge, skills, competency, and applicational ethical contact in the practice setting. (Sewell, 2018, p. 253)

As the focus of this article is on supervision within a specific practice context (statutory child and family work), the definition of the British Association of Social Workers is preferred. However, it is important to note that this definition makes no reference to ethics, unlike Sewell's definition.

\section{Method}

Six key reviews of the supervision literature were identified from the past two decades. Between them, these reviews include 250 individual articles, albeit this figure is cumulative (Table 1). The reviews were selected non-systematically and on the judgement of the author because they cover a significant period of time (the past 20 years of research), and because they focus on a range of different areas (from the functions of supervision and the supervisor-supervisee relationship, to experiences of supervision, the organisational context and outcomes for workers and people who use services). As the aim is to develop a working theory of supervision, the reviews were selected to ensure breadth, rather than following 
Table 1. Overview of the Review Articles Included in Developing the Programme Theory

\begin{tabular}{|l|c|l|}
\hline \multicolumn{1}{|c|}{ Review citation } & Articles included & \multicolumn{1}{c|}{ Areas considered } \\
\hline Tsui (1997) & 30 & $\begin{array}{l}\text { Descriptive studies, supervisory functions and the } \\
\text { supervisory relationship }\end{array}$ \\
\hline Bogo \& McKnight (2006) & 13 & $\begin{array}{l}\text { Characteristics of supervisors and the wider organisational } \\
\text { context }\end{array}$ \\
\hline Barak et al. (2009) & 27 & Worker outcomes \\
\hline Carpenter et al. (2013) & 21 & Outcomes for workers \\
\hline $\begin{array}{l}\text { O'Donoghue \& Tsui } \\
\text { (2015) }\end{array}$ & 86 & $\begin{array}{l}\text { The experience of supervision, supervision within child welfare } \\
\text { and the influence of supervision on clients and workers }\end{array}$ \\
\hline Sewell (2018) & 79 & $\begin{array}{l}\text { Reviews of research, specific models of supervision, supervisory } \\
\text { functions, experiences of supervision and worker outcomes }\end{array}$ \\
\hline
\end{tabular}

the precepts of a traditional systematic or narrative review.

The reviews were analysed to identify descriptions of how supervision works, for whom and under what circumstances. Three questions were asked in relation to each review: (1) How does supervision work and for who? (2) What helps supervision to work well and what hinders it from working well? (3) In what contexts does it work? Each review was read with these questions in mind, to produce a series of if-then statements. If-then statements are used to help understand the causal relationships that underlie the intervention (Taylor-Powell \& Henert, 2008, pp. 7-8). They are a form of mini-hypotheses about why and how something happens. Consider the following extract from Carpenter et al. (2013):

Where workers reported feeling negative about rapport, this was associated with... emotional exhaustion, whereby workers felt detached, no longer saw themselves as valuable and lost track of their personal needs. (p. 9)

From this paragraph, one can generate a series of if-then statements as follows:

IF workers feel negative about rapport with their supervisor,

THEN they will report feeling negative about rapport;
THEN they will feel emotionally exhausted and detached,

THEN they will less valued and lose track of personal needs.

Other examples of if-then statements from Carpenter et al. (2013) are given in Table 2. These statements are then grouped together to form explanatory accounts (Table 3), and those accounts in turn consolidated (Table 4 ). The same process was applied to each of the six reviews. Sheehan et al. (2018) provide a much more complete, example of a similar process in action. From these accounts, an initial programme theory was produced as both a diagram and in a descriptive format.

Table 2. Examples of Initial If-then Statements Drawn from Carpenter et al. (2013)

If workers have poor rapport with supervisors, THEN they will feel less valued

IF workers have regular contact with supervisors, THEN supervisory support becomes more important to them

IF supervisors can be relied upon when things get tough,

THEN workers are more likely to stay in their jobs

IF organisations are committed to the development of excellent supervision,

THEN workers are more likely to stay in their jobs

IF supervision is structured to enable skillsdevelopment,

THEN workers will develop their skills 
Table 3. Examples of Explanatory Accounts Drawn from Carpenter et al. (2013)

\section{Explanatory Account 1 \\ IF organisations understand the needs of their workforce, \\ THEN they can design supervision training to meet those needs \\ Explanatory Account 2 \\ IF organisations provide supervisors with suitable training, \\ THEN supervisors will develop the right skills}

\section{Explanatory Account 3}

IF organisations highlight the importance of taskassistance within supervision,

THEN supervisors will provide more taskassistance

\section{Explanatory Account 4}

IF supervisors are not given training in task-

assistance,

THEN they may not know how to do it

\section{Findings}

Within the six reviews, there were many rich ideas about how supervision works and under what circumstances. Using these ideas, an initial working theory was developed, including several contextual factors, moderators and outcomes (see Figure 1). A descriptive account of the initial programme theory can be outlined as follows:

Effective supervision is predicated on a positive working relationship between the supervisor and the worker. The supervisor will be reliable, empathic, supportive, available and knowledgeable. They will be considered authoritative because of their personal and professional qualities and as a result will be trusted and respected. The wider organisation will recognise the importance of supervision

Table 4. Consolidated accounts Drawn from Carpenter et al. (2013)

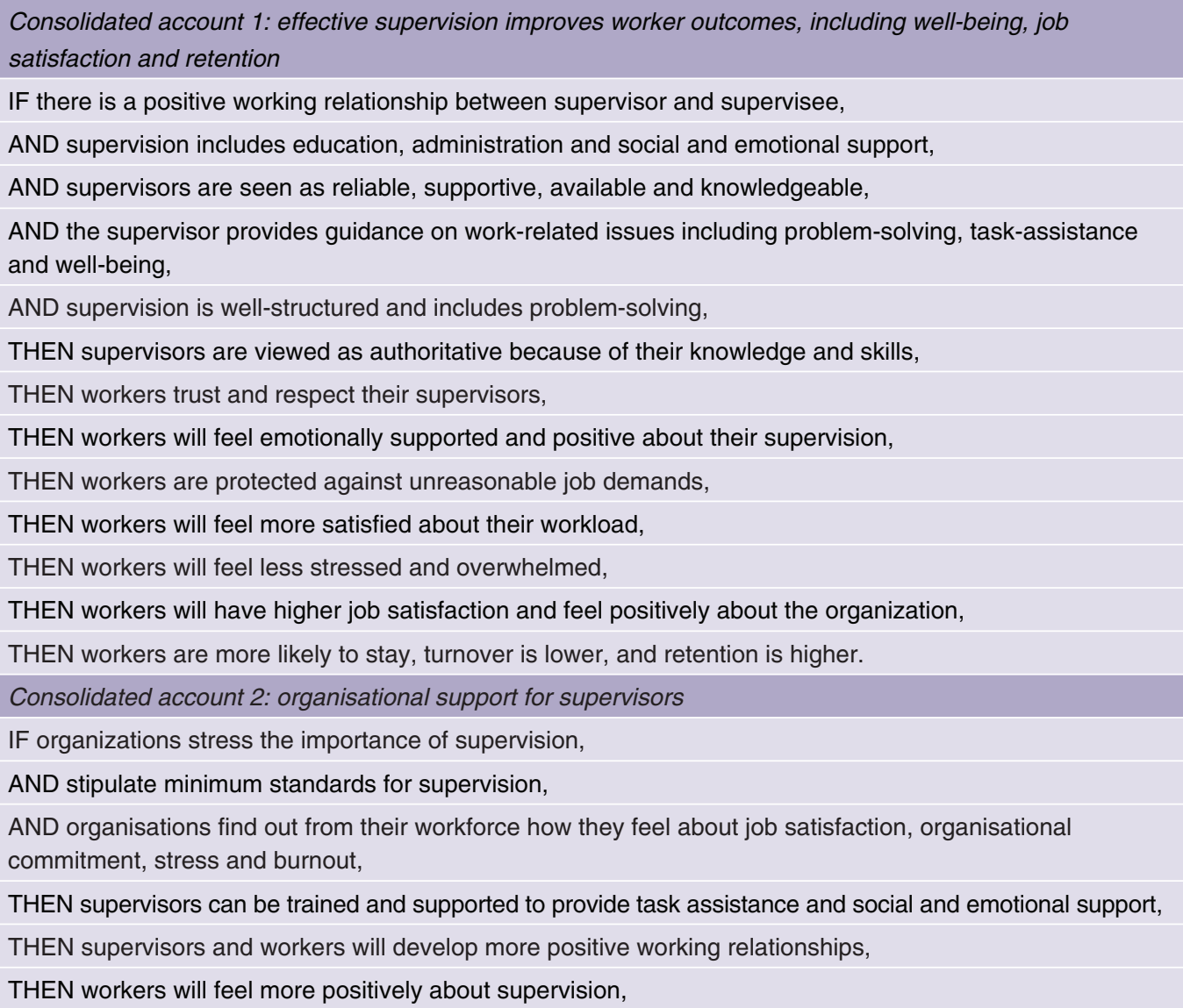


THEN supervision will be more effective,

THEN workers will develop their practice skills,

THEN workers will feel more positive about the organisation, accomplish more and experience less stress.

THEN workers will have higher job satisfaction, are more likely to stay, turnover is lower, and retention is higher.

Consolidated account 3: the benefits of supervision for the wider organisation

IF social workers feel supported by supervisors,

AND feel positive about the quality of their supervision,

THEN social workers will feel their workload is more manageable,

AND feel more emotionally satisfied at work,

AND view the wider organisation more positively.

Consolidated account 4: professional development and skills for practice

IF there is a positive working relationship between supervisor and supervisee,

AND supervisors trust social workers,

AND they share similar views about the importance of client-centred practice,

AND supervisors provide task assistance, education, training, coaching, instruction and advice,

AND help with the emotional impact of the work through reflection,

THEN workers will learn and develop their skills

AND feel more empowered and more equipped to perform their role,

AND will develop as competent professionals

Consolidated account 5: outcomes for children and families

IF there is a positive working relationship between supervisor and supervisee,

AND supervision includes education, administration and social and emotional support,

AND supervisors provide more oversight and input for complex cases,

AND supervisors help to define and describe desired outcomes for children and families,

AND provide help with problem-solving,

THEN workers will feel more empowered,

AND will develop their expertise in working with involuntary clients

AND are more likely to make better decisions

AND are more likely to work towards client-defined outcomes

AND will provide a more effective and better quality of service

and ensure it happens often and to a high standard. Supervisors will be well trained and share an understanding of workers' different needs. Supervision will be frequent and well-structured to provide education, administration and social and emotional support. The supervisor will provide guidance on work-related issues, including problemsolving, task assistance and by helping to define desirable outcomes for children and families. As a result, workers will feel emotionally supported and positive about their supervision, their supervisor and the wider organisation. Workers will be protected against unmanageable workload demands and will feel less stressed. This will ensure workers have higher rates of job satisfaction and the organisation overall will have good rates of retention and low turnover of staff. Workers will operate more autonomously, they will develop professional competence and practice skills, they will make better decisions, they will focus on client-related and client-defined outcomes and will provide a good service for children and families. 
Organisations promote the importance of supervision, stipulate minimum standards of frequency and quality, and understand the needs of their workers

Positive working relationship between

supervisor and supervisee

Supervision includes education, administration

and social / emotional support

Supervisors...
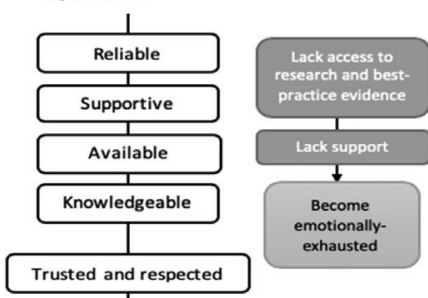

Supervision...

1

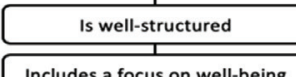

Includes a focus on well-being

Includes task-assistance

Includes task-assistance
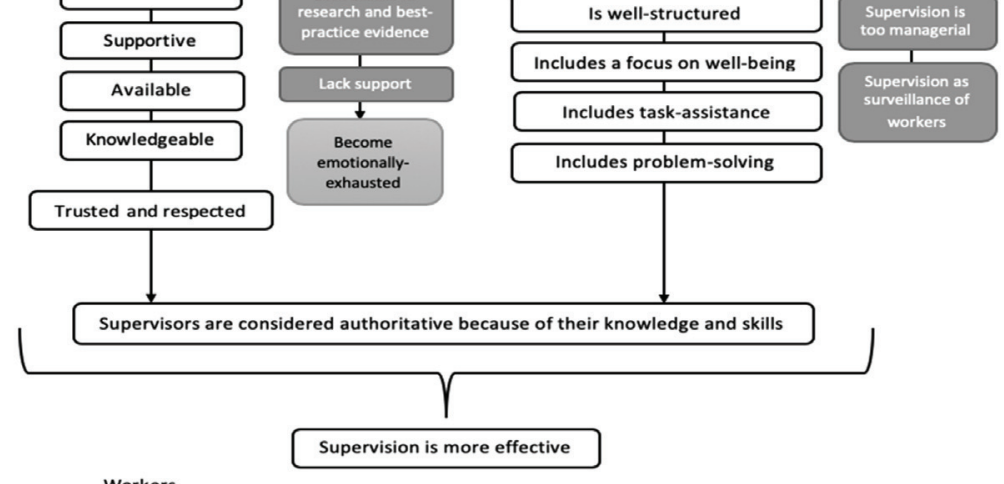

Workers...

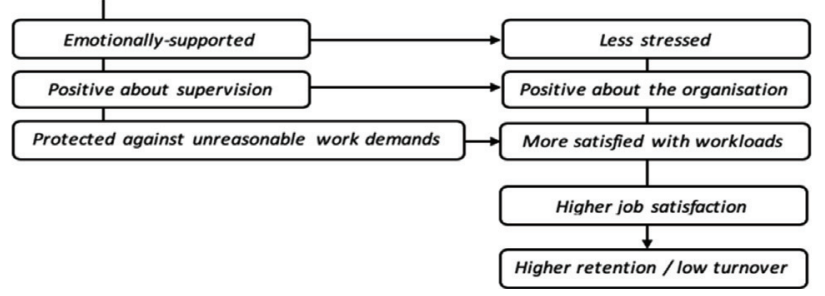

Outcomes for practice...
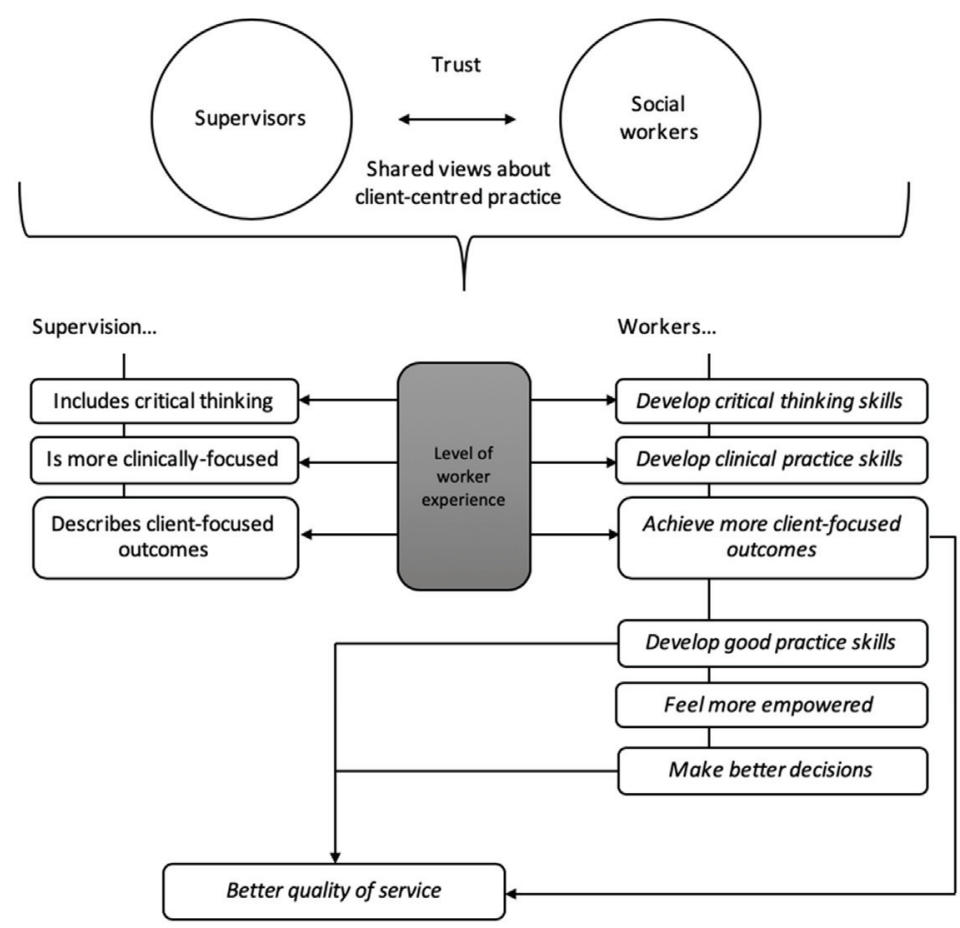

Key

\section{Context}

Positive moderators

Negative moderators

Neutral moderators

Positive outcomes

Negative outcomes

Figure 1. Initial working theory of supervision in child and family social work. 


\section{Description of key contexts, moderators and outcomes}

Key context 1 - Organisational support for supervision.

The importance of organisational support for supervision and for supervisors was perhaps surprisingly not often made explicit within the explanatory accounts, however it was suggested by enough of the individual articles (e.g., Renner, Porter, \& Preister, 2009) to be considered an important foundational context for the rest of the working theory.

Key context 2 - A positive working relationship between supervisor and supervisee.

All of the reviews were, on the other hand, very clear about the fundamental importance of a positive working relationship between supervisor and supervisee (e.g., Spence, Wilson, Kavanagh, Strong, \& Worrall, 2001). Without this context, it seems doubtful that supervision can ever be truly effective.

Key context 3 - Supervision including a range of functions.

The need to ensure supervision includes a range of functions also helps to underpin the working theory. Supervision that focuses narrowly on performance management will not be effective in a range of other ways (e.g., O’Donoghue \& Tsui, 2015).

Supervisor-related moderators - Supervisors are reliable, supportive, available, and knowledgeable.

The reviews also explored the skills and capabilities required of supervisors to provide effective supervision, including behaviours (e.g., being reliable and available: Bogo \& McKnight, 2006); attitudes (e.g. being supportive: Jacquet, Clark, Morazes, \& Withers, 2008); and professional ability (e.g., being knowledgeable about practice: Kadushin \& Harkness, 2014).

Supervision-related moderators - Supervision is well-structured, and focused on well-being, task assistance and problem-solving.
The organisation of supervision meetings was also discussed within the reviews, including what supervisors and workers should discuss in their time together. It is important that the discussion is wellstructured, (e.g., Dreyfus \& Dreyfus, 1988), that it includes time to talk about worker well-being, and that the supervisor offers help with task completion (e.g., Juby \& Scannapieco, 2007) and problem-solving (e.g., Harkness, 1995). It was also suggested that supervision should help describe and focus on client-related and client-defined outcomes (e.g., Harkness, 1995, 1997).

Worker-related outcomes - Workers feel emotionally supported, positive about supervision and protected from unreasonable work-demands.

In relation to outcomes, there was more consideration of benefits to workers (e.g., Mor Barak et al, 2009) than for people who use services. Effective supervision was considered to support workers' emotional needs (e.g., O’Donoghue \& Tsui, 2015), to contribute to more positive views of supervision and the wider organisation, and to protect workers from unreasonable work demands (as discussed particularly within Mor Barak et al.'s review, 2009). Taken together, these outcomes combine to reduce worker stress and increase retention rates.

Practice-related outcomes - Workers develop practice skills and make better decisions.

In relation to more practice-focused outcomes, there were a range of ways in which effective supervision was thought to help. Specifically, in relation to critical thinking skills (e.g., Lietz, 2008), better decision-making (e.g., Cearley, 2004) and an enhanced focus on client-defined outcomes (e.g., Harkness, 1995, 1997).

Child and family-related outcomes - A better quality of service.

The ultimate outcome of effective supervision is a better quality of service for children and families (e.g., Carpenter et al., 2013). 


\section{Discussion}

The aim of the working theory outlined above is to aid understanding of how supervision works in the context of child and family services. It may also be useful in helping to identify where there are gaps in our understanding and for planning future empirical studies, in order to test some of the different hypotheses within the model.

\section{Strengths and gaps within the theory}

The most notable strengths of the initial working theory relate to: i) the characteristics of effective supervisors and of effective supervision; and ii) worker-related outcomes. The description of an effective supervisor as being reliable, available, supportive and knowledgeable is surely one that all supervisors and workers would agree with and echoes very clearly the argument of Kadushin and Harkness (2014) that good supervisors are available, accessible, affable and able.

Similarly, the positive moderating effects of well-structured supervision that manages to provide a balanced focus on a range of different issues - including education, administration, social and emotional support, worker well-being, task assistance and problem-solving - is also likely to be very familiar and agreeable. Perhaps less familiar will be the importance of using supervision to articulate client-related, and preferably client-defined, outcomes. This aspect of the programme theory draws significantly from the work of Harkness (1987, 1995, 1997; see also Harkness \& Hensley, 1991) who found, in one of the few experimental studies of supervision, that a more outcomes-focused approach in supervision predicted client satisfaction with the service. Worker-related outcomes are also well described in the programme theory, with links made between the kinds of supervisor and supervisory characteristics outlined above and more manageable workloads, reduced levels of stress, a greater sense of empowerment, greater job satisfaction and higher retention rates. The logic of the relationships between these different elements is clear. Yet there are also some gaps in the theory as it currently stands, primarily: i) worker-related moderators; and ii) outcomes for children and families.

The programme theory outlines the key characteristics of effective supervisors (and of effective supervision sessions). Yet other than one neutral moderator in relation to worker experience, it contains limited details about the worker. This suggests that supervision will be effective dependent on the context and supervisor-related moderators, but independently of how the worker behaves and responds to it - a clearly unsustainable proposition. There are no complex social interventions which do not depend on how people behave and respond to them - and supervision is no different. Besides different levels of experience, what other workerrelated factors might be important?

First, the worker's personal and professional values. There is one reference in the programme theory to the need for supervisors and workers to share a belief in the value of client-centred practice. Yet there are many other aspects of the worker's personal and professional value base we might also want to consider. For example, how far do they believe that genuine collaboration with parents is possible in the context of child protection services and when the risk to the child is high (Whittaker \& Wilkins, 2018)? For that matter, what are the supervisor's values and beliefs in this regard? In the absence of these kinds of shared values, it seems unlikely that time spent in supervision describing and defining client-related outcomes would be of much use. Second, the worker's current practice skills and knowledge base. It may be that experience is a useful correlate of worker skill but there is not necessarily a direct link between the two. As noted by McHugh and Lake (2010, p. 279), "[e]xperience is a necessary but not sufficient condition for expertise and not all experienced [practitioners] are experts". Third, the worker's personal motivation 
and enthusiasm for the work (separate from their personal values). If we consider the educational function of supervision, it has long been recognised in other settings that the motivation of the learner is a key moderator for the transfer of knowledge from "classroom" to practice (Gegenfurtner \& Vauras, 2012). All of these issues suggest that we have further theorising to do about the role of the worker in supervision. We may know a great deal about what makes for an effective supervisor, yet we know comparatively little about what makes for an effective supervisee.

The question of outcomes for people who use services is also relatively undefined within the initial programme theory. As in many fields of practice, the question of outcomes in child and family social work is far from straightforward. It has been argued that we have become too focused on outcomes, failing to recognise that children's services are primarily rightsbased, and do not necessarily aim to improve (measurable) outcomes in the majority of cases (Forrester, 2019). That being said, the relationship between practice and outcomes is also both more complicated and less significant than it first appears (Forrester et al, 2019). For most families referred to statutory services, social work makes little difference (Forrester et al., 2019). Not because social workers are poor at their jobs, but because most families do not have the kind of long-standing, serious problems that could benefit from social work involvement (Forrester et al., 2019). Within the initial programme theory as it stands, there is a greater emphasis on improving practice skills as a mechanism for improving outcomes than there is on protecting and promoting human rights. If it is true that social work is primarily a rights-based profession (Schraer, 2014), then effective supervision must have an important part to play in ensuring that workers are able to fulfil this central aspect of the role. A more refined version of the programme theory would therefore need to articulate more fully not only what a better service means in terms of measurable outcomes, but to ensure this includes protection and promotion of human rights.

\section{Social work supervision as a moral and technical activity}

It has long been considered that social work is both a rational-technical activity and a practical-moral one (Jordan, 1978) - and the same is true of supervision. When it is concerned with management accountability or even the surveillance of practice (Beddoe, 2010), supervision is overtly technical in nature. Yet supervisors also have a shared responsibility to set the moral climate for practice and to consider how social workers can be supported to "do the right thing" rather than (technically) to "do things right" (Munro, 2011). In the complex context of child and family services, where competing rights are balanced (Carter, 2016), identifying the right thing to do often requires some negotiation - and supervision can be a key forum for such negotiations to take place. Yet this is not reflected in the initial version of the working theory. This aspect of supervision is likely to form an important part in any future development of the working theory.

\section{The absence of risk}

A similar argument can be made in relation to the absence of risk. This may be suitable for supervision more generically but, in relation to statutory child and family services, it is a notable missing component, especially from the perspective of practitioners. As with the moral and rights-based dimensions of supervision, the relationship between risk and supervision is likely to form part of any future development.

\section{Comparing the programme theory to the evidence-informed social work supervision model: similar yet different}

Finally in this section, how does the working theory compare to other approaches in the same field, specifically O'Donoghue, Wong Ju, and Sui's (2018) evidence-informed supervision model? This model was, similar to the working 
theory presented here, "pragmatically constructed...from research findings from social work supervision research" (p. 348), with the aim of "strengthening social work supervision...by building on the work of those who have gone before" (p. 354). The model contains five elements the construction of supervision (how it is conceived and practised); supervision of practitioners (e.g., social and emotional support); the supervisory relationship, interactional processes (e.g., structure and the dynamics of the process); and the supervision of practice (e.g., clinical supervision). Compared to the working theory in this article, it focuses more on the evidence for each of these components and less on how different mechanisms and moderators combine to create desirable (or undesirable) outcomes.

\section{Strengths and limitations of the study}

The main strengths of this article are the attempt to bring together six significant reviews of the social work supervision literature in order to combine the underlying ideas about how and why supervision works within the context of child and family services.

As with any article, there are also some limitations. The working theory would have been developed in more detail had it relied on individual articles, rather than reviews. There are also many other articles on supervision that were not included within the reviews, and therefore have not influenced the theory. This is particularly true of non-empirical work. The working theory would also be enhanced via consultation with supervisors and supervisees and it is intended for this to happen in the near future.

\section{Using the theory for further research}

Realist evaluation "starts with a theory and ends with a more refined theory" (Currie et al., 2019, p. 1322). To refine this initial version of the theory, two studies are being planned. The first involves consultation with a range of supervisors and practitioners to ask whether the theory makes sense to them and what adaptations might be needed for specific contexts. For example, how do supervisors adapt their approach depending on the experience of the worker and how can this be reflected within the theory? Secondly, by breaking down the complex intervention of supervision into more manageable parts, we increase the scope of evaluative researchers to test the embedded if-then hypotheses. Evaluating the influence of supervision on people who use services is challenging, not least because of the high number of variables that would need to line up in order to establish anything like a direct effect (Fleming \& Steen, 2004). By using this working theory, elements within this web of variables can be evaluated more directly. For example, what empirical evidence can we find to support the assertion that workers who feel more positive about supervision also feel more positive about the wider organisation? We know from existing studies that the assertion is at least partially supported by the evidence - but what are the moderators that affect the relationship and are there situations in which the relationship does not operate? Similarly, if supervisors do focus more on client-defined outcomes within supervision, is it true (in this context) that more client-defined outcomes are achieved? For this latter example, there is currently a study ongoing in England to explore exactly this question.

\section{Conclusion}

Developing a working theory is both the first and last step within the realist approach to evaluation. Initial theories such as this one can be refined in a number of ways, including by engagement with experts (e.g., supervisors and workers) and by empirical study. As outlined above, the intention is to do both of these things, to refine the theory to the point where it provides not only a more complete description of the intervention but also a helpful template for good supervisory practice within the child and family social work context. 


\section{References}

Baginsky, M., Moriarty, J., Manthorpe, J., Stevens, M., Maclnnes, T., \& Nagendran, T. (2010). Social workers workload survey: Messages from the frontline. London, UK: Department for Children, Schools and Families.

Beddoe, L. (2010). Surveillance or reflection: Professional supervision in "the risk society." British Journal of Social Work, 40(4), 1279-1296.

Beddoe, L., Karvinen-Niinikoski, S., Ruch, G., \& Tsui, M (2015). Towards an international consensus on a research agenda for social work supervision: Report on the first survey of a Delphi study. British Journal of Social Work, 46(6), 1568-1586. doi:10.1093/bjsw/bcv110

Bogo, M., \& Mcknight, K. (2006). Clinical supervision in social work. The Clinical Supervisor, 24(1-2), 49-67. doi:10.1300/j001v24n01_04.

Bostock, L., Forrester, D., Patrizo, L., Godfrey, T., \& Zonouzi, M. (2017). Scaling and deepening the reclaiming social work model: Evaluation report. London, UK: Department for Education.

Bostock, L., Patrizo, L., Godfrey, T., Munro, E., \& Forrester, D. (2019). How do we assess the quality of group supervision? Developing a coding framework. Children and Youth Services Review, 100, 515-524. doi:10.1016/j.childyouth.2019.03.027

British Association of Social Workers. (2011). UK supervision policy. Retrieved from http://cdn.basw.co.uk/upload/ basw_73346-6.pdf

Byng, R., Norman, I., \& Redfern, S. (2005). Using realistic evaluation to evaluate a practice-level intervention to improve primary healthcare for patients with long-term mental illness. Evaluation, 11(1), 69-93. doi:10.1177/1356389005053198

Byng, R., Norman, I., Redfern, S., \& Jones, R. (2008). Exposing the key functions of a complex intervention for shared care in mental health: Case study of a process evaluation. BMC Health Services Research, 8(1). doi:10.1186/1472-6963-8-274

Carpenter, J., Webb, C. M., \& Bostock, L. (2013). The surprisingly weak evidence base for supervision: Findings from a systematic review of research in child welfare practice (2000-2012). Children and Youth Services Review, 35(11), 1843-1853. doi:10.1016/j. childyouth.2013.08.014

Carter, R. (2016). Social work is about human rights, not assessing the life out of people. Retrieved from https://www.communitycare.co.uk/2016/07/06/socialwork-human-rights-assessing-life-people/

Cashwell, C. S., Looby, E. J., \& Housley, W. F. (1997). Appreciating cultural diversity through clinical supervision. The Clinical Supervisor, 15(1), 75-85. doi:10.1300/j001v15n01_06

Cearley, S. (2004). The power of supervision in child welfare services. Child and Youth Care Forum, 33(5), 313-327.

Collins-Camargo, C., \& Royse, D. (2010). A study of the relationships among effective supervision, organizational culture promoting evidence-based practice, and worker self-efficacy in public child welfare. Journal of Public Child Welfare, 4(1), 1-24. doi:10.1080/15548730903563053

Currie, J., Chiarella, M., \& Buckley, T. (2019). Realist evaluation of privately practising nurse practitioners in Australia: Development and refinement of theories. Collegian, 26(1), 8-15. doi:10.1016/j. colegn.2018.01.006

Davys, A. (2017). Interprofessional supervision: A matter of difference. Aotearoa New Zealand Social Work, 29(3), 79-94.

Davys, A., May, K., Burns, B., \& O’Connell, M. (2017). Evaluating social work supervision. Aotearoa New Zealand Social Work, 29(3), 108-121.

Dreyfus, H. L., \& Dreyfus, S. E. (1988). Mind over machine: The power of human intuition and expertise in the era of the computer. New York, NY: Free Press.

Elkington, J. (2014). A kaupapa Māori supervision context cultural and professional. Aotearoa New Zealand Social Work, 26(1), 65-73.

Fleming, I., \& Steen, L. (2013). Supervision and clinical psychology theory, practice and perspectives. Hoboken, NJ: Taylor and Francis.

Forrester, D. (2019, May). What is the point of child and family social work? Retrieved from https://sites.cardiff. ac.uk/cascade/2019/05/15/what-is-the-point-of-child-andfamily-social-work/

Forrester, D., Westlake, D., Killian, M., Antonopolou, V., McCann, M., Thurnham, A., ... Hutchison, D. (2019). What is the relationship between worker skills and outcomes for families in child and family social work? The British Journal of Social Work. doi:10.1093/ bjsw/bcy 126

Funnell, S. C., \& Rogers, P. J. (2011). Purposeful program theory effective use of theories of change and logic models. San Francisco, CA: Jossey-Bass.

Gegenfurtner, A., \& Vauras, M. (2012). Age-related differences in the relation between motivation to learn and transfer of training in adult continuing education. Contemporary Educational Psychology, 37(1), 33-46. doi:10.1016/j.cedpsych.2011.09.003

Harkness, D. R. (1987). Social work supervision in community mental health: Effects of normal and client-focused supervision on client satisfaction and generalized contentment (Unpublished doctoral dissertation). University of Kansas. Ann Arbor, Ml: University Microfilms International.

Harkness, D. (1995). The art of helping in supervised practice. The Clinical Supervisor, 13(1), 63-76. doi:10.1300/j001v13n01_05

Harkness, D. (1997). Testing interactional social work theory. The Clinical Supervisor, 15(1), 33-50. doi:10.1300/j001v15n01_03

Harkness, D., \& Hensley, H. (1991). Changing the focus of social work supervision: Effects on client satisfaction and generalized contentment. Social Work, 36(6), 506-512. doi:10.1093/sw/36.6.506

Jacquet, S. E., Clark, S. J., Morazes, J. L., \& Withers, R. (2008). The role of supervision in the retention of public child welfare workers. Journal of Public Child Welfare, 1(3), 27-54.

Jordan, B. (1978). A comment on "Theory and Practice in Social Work." British Journal of Social Work, 8(1), 23-25. doi.org/10.1093/oxfordjournals.bjsw.a056919

Juby, C., \& Scannapieco, M. (2007). Characteristics of workload management in public child welfare agencies. Administration in Social Work, 31(3), 95-109. 
Kadushin, A., \& Harkness, D. (2014). Supervision in social work (fifth edition). New York, NY: Columbia University Press.

Lees, A. (2017). Evaluation of reflective practice groups project: Brighton and Hove Children's Services. London, UK: Centre for Social Work Practice.

Lietz, C. A. (2008). Implementation of group supervision in child welfare: Findings from Arizona's supervision circle project. Child Welfare, 87(6), 31-48.

Manthorpe, J., Moriarty, J., Hussein, S., Stevens, M., \& Sharpe, E. (2015). Content and purpose of supervision in social work practice in England: Views of newly qualified social workers, managers and directors. British Journal of Social Work, 45(1), 52-68. doi:10.1093/bjsw/bct102

McHugh, M., \& Lake, E. (2010). Understanding clinical expertise: Nurse education, experience, and the hospital context. Research in Nursing and Health, 33(4), 276-287.

Moorhouse, L., Hay, K., \& O’Donoghue, K. (2014). Listening to student experiences of supervision. Aotearoa New Zealand Social Work, 26(4), 37-52.

Mor Barak, M. M., Travis, D., Pyun, H., \& Xie, B. (2009). The impact of supervision on worker outcomes: A meta-analysis. Social Service Review, 83(1), 3-32. doi: $10.1086 / 599028$

Munro, E. (2011). The Munro review of child protection: Final report. Retrieved from https://assets.publishing. service.gov.uk/government/uploads/system/uploads/ attachment_data/file/175391/Munro-Review.pdf

O'Donoghue, K., Wong Yuh Ju, P., \& Tsui, M.-S. (2018). Constructing an evidence-informed social work supervision model. European Journal of Social Work, 21(3), 348-358.

O'Donoghue, K., \& Tsui, M. (2015). Social work supervision research (1970-2010): The way we were and the way ahead. British Journal of Social Work, 45(2), 616-633. doi:10.1093/bjsw/bct115

Pawson, R., \& Tilley, N. (2014). Realistic evaluation. Los Angeles, CA: Sage.

Rankine, M. (2017). Making the connections: A practice model for reflective supervision. Aotearoa New Zealand Social Work, 29(3), 66-78.

Renner, L., Porter, R., \& Preister, S. (2009). Improving the retention of child welfare workers by strengthening skills and increasing support for supervisors. Child Welfare, 88(5), 109-127.

Schraer, R. (2014, July 23). "Social work is a human rights discipline": IFSW president speaks up for the profession. Retrieved from https://www.communitycare. co.uk/2014/07/23/social-work-human-rights-disciplineifsw-president-speaks-profession/

Schraer, R. (2016, January 27). Patchy implementation of the ASYE leaves newly qualified social workers facing unprotected caseloads. Retrieved from http://www.communitycare.co.uk/2016/01/27/patchyimplementation-asye-leaves-newly-qualified-socialworkers-facing-unprotected-caseloads/

Sewell, K. M. (2018). Social work supervision of staff: A primer and scoping review (2013-2017). Clinical Social Work Journal, 46(4), 252-265. doi:10.1007/ s10615-018-0679-0
Shearn, K., Allmark, P., Piercy, H., \& Hirst, J. (2017). Building realist program theory for large complex and messy interventions. International Journal of Qualitative Methods, 16(1), 1-11. doi:10.1177/1609406917741796

Sheehan, L., O'Donnell, C., Brand, S., Forrester, D., Addis, S., El-Banna, A., Kemp, A., \& Nurmatov, U. (2018, November). Signs of safety: Findings from a mixedmethods systematic review focussed on reducing the need for children to be in care. Retrieved from https://s29720.pcdn.co/wp-content/uploads/Signs_of_ Safety_a_mixed_methods_systematic_review.pdf

Smith, C., Russell, R., \& Giddings, M. (2007). Evaluating a social work supervision model in a real-world child welfare setting. Professional Development: The International Journal of Continuing Social Work Education, 10(1), 10-24.

Spence, S. H., Wilson, J., Kavanagh, D., Strong, J. \& Worrall, L. (2001). Clinical supervision in four mental health professions: A review of the evidence. Behaviour Change, 18(3), 135-155.

Taylor-Powell, E., \& Henert, E. (2008). Developing a logic model: Teaching and training guide. Retrieved from https://fyi.extension.wisc.edu/programdevelopment/files/ 2016/03/Imguidecomplete.pdf

Tsui, M. (1997). Empirical research on social work supervision. Journal of Social Service Research, 23(2), 39-54. doi:10.1300/j079v23n02_03

Turner-Daly, B., \& Jack, G. (2014). Rhetoric vs. reality in social work supervision: The experiences of a group of child care social workers in England. Child \& Family Social Work, 22(1), 36-46. doi:10.1111/cfs.12191

Whittaker, C., \& Wilkins, D. (2018). Doing child protection social work with parents: What are the barriers in practice? British Journal of Social Work, 48(7), 2003-2019.

Wilkins, D., Forrester, D., \& Grant, L. (2017). What happens in child and family social work? Child and Family Social Work, 22(2), 942-951. 